\title{
Abnormal coronary artery angiography is not associated with adverse pregnancies outcomes
}

\author{
Margriet A. M. Heemskerk ${ }^{1,2}$, Hemanth Ramanna ${ }^{1}$, Kitty W. M. Bloemenkamp ${ }^{3}$, \\ Christianne J. M. de Groot ${ }^{2}$
}

${ }^{1}$ Department of Cardiology, Medical Centre Haaglanden, Hague, The Netherlands

${ }^{2}$ Obstetrics and Gynecology, VU University Medical Centre, Amsterdam, The Netherlands

${ }^{3}$ Department of Obstetrics, Leiden University Medical Centre, Leiden, The Netherlands

Email: cdegroot@me.com

Received 18 November 2011; revised 19 December 2011; accepted 14 January 2012

\section{ABSTRACT}

Background: Recently, epidemiologic studies have suggested an association between pregnancy complications and the development of coronary artery disease later in life. The current study investigate the relation between obstructive coronary versus normal coronary angiographies and the prevalence of complications during pregnancy including preeclampsia, pregnancy induced-hypertension, low birth weight, and preterm birth. Methods: All consecutive women aged $<55$ year, who had coronary angiography were included. Based on angiography outcome, patients were classified as normal (controls: no stenosis, wall irregularity without stenosis, or minimal calcification present) or abnormal (subjects). A standard questionnaire was sent to all participating women. The questionnaires inquired into risk factors for coronary vascular disease, in particular history of hypertensive diseases, and specific obstetric history. The results were analyzed using the unpaired $t$-test, the Chi-squared test, and Pearson's correlation coefficients as appropriate. A significance level of $\mathbf{P}<\mathbf{0 . 0 5}$ was used. Results: Of the 211 consecutive women aged $<55$ years, $62 \%(n=131)$ had normal coronary angiography and $38 \%(n=80)$ abnormal coronary angiography. We found no differences of reported pregnancy complications including preeclampsia, hypertension, low birth weight, and preterm birth (combined $R R=0.70$, $95 \%$ C.I. $=0.38-1.3$ ) in case of abnormal coronary angiography compared to women with normal coronary angiography. The traditional risk factors hypercholesterolemia, tobacco use, and diabetes were identified as the major risk factors for developing cardiovascular disease, with a relative risk ranging from 4.2 1.8. Conclusion: These data suggest that pregnancy complications are not an important denominator for cardiovascular disease.
Keywords: Coronary Artery Angiography; Pregnancy; Preeclampsia

\section{INTRODUCTION}

Cardiovascular disease is one of the most common causes of disability and early death in the developed world. The underlying mechanism of this disease, arteriosclerosis, is often not recognized until the onset of symptoms. Mass screening for cardiovascular disease has not been proven to be a cost-effective way to prevent disease. Secondary prevention, however, is effective, although the magnitude of its effect remains quite uncertain [1].

It has been suggested that women who had a pregnancy complicated by preeclampsia have a higher risk to develop cardiovascular disease later in life $[2,3]$. Preeclampsia is a human-pregnancy-specific syndrome that is considered a leading cause of maternal and perinatal morbidity and mortality. The prevalence is $3 \%-5 \%$ of pregnancies in the developed world. The characteristic clinical findings are hypertension and proteinuria [4].

Risk factors for developing preeclampsia include dyslipidaemia, insulin resistance, hypercoagulability, and inflammation during pregnancy. Most of these factors are also well-known risk factors for cardiovascular disease [3]. Therefore, it is unclear whether preeclampsia increases the chance of developing cardiovascular disease in later life or that preeclampsia is a pre-existing risk factor that manifests during pregnancy. The latter hypothesis suggests that pregnancy is a stress test for diseases in later life $[5,6]$.

Previous studies enrolled women with preeclampsia as selection criterion, when studying the association of cardiovascular disease in later life [7-10]. In contrast, we have analyzed women with cardiovascular disease as selection criteria defined by abnormal coronary an-giography. We hypothesized that middle-aged women with abnormal coronary angiography have an increased risk of complica- 
tions during pregnancy including preeclampsia, pregnancy induced-hypertension, low birth weight, and preterm birth compared to women with normal coronary angiography. Additionally, we have identified risk factors for cardiovascular risk in women with abnormal coronary angiography.

\section{PATIENTS AND METHODS}

All consecutive women aged $<55$ year, who had coronary angiography at Medical Center Haaglanden, The Hague between January $1^{\text {st }}, 2005$ through October $1^{\text {st }}, 2007$ were included. Based on angiography outcome, patients were classified as normal (controls) or abnormal (subjects). A coronary angiography was graded normal when no stenosis, wall irregularity without stenosis, or minimal calcification was present. Indication for angiography was acute coronary syndrome (3), chest pain with and without abnormal EKG (182), Left ventrikel dysfunction (23) and valve abnormality (3).

The patients' weight and height, serum creatinine, and fasting levels of triglycerides were recorded at coronary angiography. High body mass index, high serum creatinine concentrations, and high fasting levels of triglycerides were defined respectively $25 \mathrm{~kg} / \mathrm{m}^{2}, 100 \mu \mathrm{mol} / \mathrm{l}$, and 1.7 $\mathrm{mmol} / \mathrm{l}[7]$.

Additionally, blood pressure and fasting levels of glucose and total plasma cholesterol were recorded, as were medication for hypertension, diabetes, and hypercholesterolemia. Hypertension was defined as a systolic blood pressure greater than $140 \mathrm{mmHg}$ and/or diastolic blood pressure greater than $85 \mathrm{mmHg}$, or use of antihypertensive medication [1]. Diabetes was defined as fasting level of glucose greater than $6.0 \mathrm{mmol} / 1$ or use of anti diabetic medication. Hypercholesterolemia was defined as plasma total cholesterol greater than $5.0 \mathrm{mmol} / \mathrm{l}$ or a normal value by use of cholesterol lowering agents including statin therapy [1].

\section{Questionnaires}

A standard questionnaire was sent to all participating women and followed by an additional telephone call if necessary. The questionnaires inquired into risk factors for coronary vascular disease, in particular history of hypertensive diseases, and specific obstetric history. The risk factors for cardiovascular disease included smoking habits and family history for cardiovascular disease [11].

Smoking history was defined positive when the subject had a smoking habit for more than 5 years over the last twenty years. Family history for cardiovascular disease was defined positive when she had a first-degree family member younger than 65 years, with at least one of the following diseases: hypertension, diabetes, hypercholesterolemia, myocardial infarction, stroke, thrombo- sis, or pulmonary embolism.

The obstetric questionnaires surveyed specific risk factors for developing preeclampsia, such as blood pressure, and additional complications during the pregnancies [12], including low birth weight. Preeclampsia was defined by the novo hypertension after the $20^{\text {th }}$ week of gestation, associated with proteinuria $[13,14]$.

Further specific pregnancy data were acquired, including gestational age, birth weight, and parity. Low birth weight of the child was defined as a birth weight of less than 2500 gram in at least one of the children. Premature birth was defined as at least one delivery before $<37$ weeks.

If follow-up data could not be obtained by questionnaire and telephone, the General Practitioner was queried. A total of 303 consecutive women who were identified eligible for this study. Of these women, 211 (70\%) were willing to participate.

Of the 92 non-respondents, 5 were deceased ( 1 died of autoimmune disease, 2 of cardiovascular disease, and 2 of unknown causes), 25 women were not willing to participate for several reasons, including having no time, no children or no interest.

40 women could not be traced; they had an unknown address, an unknown phone number or left the last known general practitioner. In addition, for 22 women, the general practices were unable to provide data.

The results of the participants were analyzed in cross tables. Comparisons were made using the unpaired $t$-test, the Chi-squared test, and Pearson's correlation coefficients as appropriate. A significance level of $\mathrm{P}<0.05$ was used. We calculated that a total of 200 women were needed to find a two-and-a-half-fold difference between the groups with alpha of 0.05 and power of 0.80 .

\section{RESULTS}

Of the 211 participating women, $131(62 \%)$ showed a normal angiography (controls) and 80 (38\%) showed an abnormal angiography (subjects). Biochemical, demographic data, and risk factors for developing cardiovascular disease are shown in Table $\mathbf{1}$.

Women with an abnormal coronary angiography on average were older than women with a normal angiography and had higher fasting serum triglycerides and higher fasting serum glucose and more often hypercholesterolemia.

Data regarding their pregnancies are described in Table 2. No differences were found in the prevalence of pregnancy complications including preeclampsia, hypertension (hypertensive disorders $\mathrm{RR}=0.71$, 95\% C.I. $=$ 0.38 - 1.39), low birth weight, and preterm birth (combined $\mathrm{RR}=0.70,95 \%$ C.I. $=0.38-1.3$ ) between women with a normal angiography and women with abnormal angiography. Women with abnormal angiography indicated a higher tendency to smoke during pregnancies $(\mathrm{RR}=1.8,95 \%$ C.I. $=0.9$ - 3.6). 
Table 1. Demographics and risk factors for developing cardiovascular disease.

\begin{tabular}{llll}
\hline Riskfactor (normal values) & Abnormal angiography $(\mathrm{n}=80)$ & Normal angiography $(\mathrm{n}=131)$ & P-value \\
\hline Age in years, mean $\pm \mathrm{SD}$ & $52.3 \pm 4.6$ & $48.9 \pm 6.2$ & $<0.001$ \\
BMI $\left(<25 \mathrm{~kg} / \mathrm{m}^{2}\right)$, mean $\pm \mathrm{SD}$ & $29.7 \pm 5.01$ & $28.3 \pm 5.7$ & 0.08 \\
Serum creatinine $(<100 \mu \mathrm{mol} / \mathrm{l})$, mean $\pm \mathrm{SD}$ & $87.7 \pm 101.0(101.0)$ & $73.3 \pm 15.2$ & 0.13 \\
Triglycerides $(<1.7 \mathrm{mmol} / \mathrm{l})$, mean $\pm \mathrm{SD}$ & $2.0 \pm 1.1$ & $1.6 \pm 1.1$ & 0.03 \\
Fasting glucose $(<6.0 \mathrm{mmol} / \mathrm{l})$, mean $\pm \mathrm{SD}$ & $7.1 \pm 2.8$ & $6.2 \pm 1.7$ & 0.05 \\
Plasma cholesterol $(<5.0 \mathrm{mmol} / \mathrm{l})$, mean $\pm \mathrm{SD}$ & $4.9 \pm 1.3$ & $5.0 \pm 1.1(1.1)$ & 0.63 \\
Hypertension $(\%)$ & $53(66 \%)$ & $66(50 \%)$ & 0.07 \\
Diabetes Mellitus $(\%)$ & $34(43 \%)$ & $33(25 \%)$ & 0.01 \\
Hypercholesterolemia $(\%)$ & $52(65 \%)$ & $35(27 \%)$ & $<0.001$ \\
Smoking $>5$ years $(\%)$ & $44(55 \%)$ & $50(21 \%)$ & 0.06 \\
Positive family history of CVD $(\%)$ & $67(84 \%)$ & $96(73 \%)$ & 0.08 \\
\hline
\end{tabular}

Data are expressed as, means \pm SD and number (\%).

Table 2. Obstetric outcomes of the pregnancies (percentages in parentheses).

\begin{tabular}{|c|c|c|c|}
\hline & Abnormal angiography $(\mathrm{n}=80)$ & Normal angiography $(\mathrm{n}=131)$ & RR ( $95.0 \%$ C.I. $)$ \\
\hline Average number of children, mean $\pm \mathrm{SD}$ & $2.4 \pm 1.4$ & $2.2 \pm 1.5$ & \\
\hline Average maternal age at $1^{\text {st }}$ born mean $\pm \mathrm{SD}$ & $23.9 \pm 5.4$ & $23.6 \pm 5.5$ & \\
\hline Preeclampsia during $1^{\text {st }}$ pregnancy $(\%)$ & $2(2.5 \%)$ & $5(3.8 \%)$ & $0.96(0.50-1.87)$ \\
\hline Preeclampsia during any pregnancy (\%) & $5(6.3 \%)$ & $9(6.9 \%)$ & $0.90(0.29-2.80)$ \\
\hline Hypertension during $1^{\text {st }}$ pregnancy $(\%)$ & $12(15 \%)$ & $25(19.1 \%)$ & $0.68(0.41-1.15)$ \\
\hline Hypertension during any pregnancy (\%) & $15(18.8 \%)$ & $35(26.7 \%)$ & $0.63(0.32-1.25)$ \\
\hline Birth weight child $<2500 \operatorname{gr}(\%)$ & $8(10 \%)$ & $18(13.7 \%)$ & $0.71(0.29-1.76)$ \\
\hline Preterm birth (\%) & $8(10 \%)$ & $14(10.1 \%)$ & $0.94(0.37-2.37)$ \\
\hline Total number of spontaneous abortion $\geq 3(\%)$ & $4(5.0 \%)$ & $10(7.6 \%)$ & $0.68(0.19-2.10)$ \\
\hline Smoking during pregnancy (\%) & $21(26.3 \%)$ & $22(16.8 \%)$ & $1.78(0.88-3.59)$ \\
\hline
\end{tabular}

Data expressed as means $\pm \mathrm{SD}$, number (\%) as appropriate and relative risk (RR) with $95 \%$ confidence interval (95\% C.I.).

Women with an abnormal coronary angiography had more often hypertension $(\mathrm{RR}=1.78,95 \%$ C.I. $=1.0$ $3.1)$, diabetes $(\mathrm{RR}=2.2,95 \%$ C.I. $=1.2-3.9)$ and hypercholesterolemia $(\mathrm{RR}=5.1,95 \%$ C.I. $=2.8-9.3)$ at time of coronary angiography. In addition, women with abnormal coronary angiography smoked more often than women with a normal coronary angiography $(\mathrm{RR}=1.9$, $95 \%$ C.I. $=1.1-3.5$ ).

Using multivariate regression model, overall, an abnormal angiography was associated independently with hypercholesterolemia $(\mathrm{RR}=3.2,95 \%$ C.I. $=1.5-6.7)$ and smoking $(\mathrm{RR}=2.9,95 \%$ C.I. $=1.3-6.5)$ and not with pregnancy complications (i.e. preeclampsia $\mathrm{RR}=$ $1.1,95 \%$ C.I. $=0.50-2.5$ ), pregnancy-induced hypertension $(R R=0.57,95 \%$ C.I. $=0.29-1.1)$, low birth weight $(\mathrm{RR}=0.72,95 \%$ C.I. $=0.23-2.3)$ and preterm birth $(\mathrm{RR}$ $=0.77,95 \%$ C.I. $=0.34-1.7)$. For all pregnancy complications combined, $\mathrm{RR}=0.68,95 \%$ C.I. $=0.33$ - 1.4.

\section{DISCUSSION}

The present study found no differences in prevalence of pregnancy complications in women with cardiovascular disease defined by abnormal coronary angiography compared to women with normal coronary angiography. Preeclampsia, hypertension during pregnancy, low birth weight of the child, and preterm birth were not associated with abnormal coronary angiography, neither as separate nor as combined complications. Identified risk factors for cardiovascular risk in women with abnormal coronary angiography were the classic risk factors including hypercholesterolemia and smoking.

Our data are in contrast with recent epidemiologic studies indicating women with a history of preterm preeclampsia have an eightfold risk for death from cardiovascular causes as compared with women who had normal pregnancies. From these data, the investigators con- 
cluded that factors that increase the risk for cardiovascular disease might also be linked to preeclampsia [15]. This was further supported by the finding that classic risk factors for cardiovascular disease in women with a history of preeclampsia including systolic and diastolic blood pressure, body mass index, and concentrations of cholesterol and triglycerides [10,16,17] were increased compared to women with uncomplicated pregnancies. In addition, Blaauw et al. [12] found increased intima-media thickness (IMT) of femoral arteries measured by ultrasonography in women who had preeclampsia three months post partum. Intima-media thickness is a generally accepted marker of preclinical atherosclerosis and therefore associated with cardiovascular disease.

Differences between previous studies and our results can possible be explained by differences in the cohort of women studied; in the former studies women with and without preeclampsia were studied in prospective epidemiological studies for cardiovascular disease in later life whereas we studied women who had coronary angiography and were questioned for pregnancy complications earlier.

However, Valdes et al. [18] did demonstrate that women with hypertension during pregnancies have earlier coronary disease proved with an angiography. However, Valdes et al compared women with hypertension during pregnancy and women with uncomplicated pregnancies. Although, a description of adverse pregnancy outcomes and exact definition of hypertensionare lacking.

Haukkamaa et al. [19] employed a similar study design as described in our study. For women $<66$ years with abnormal coronary angiography, they found a five-fold increased risk of preeclampsia. Haukkamaa et al. [19] described a prevalence of $21 \%$ women with preeclampsia of those with abnormal coronary angiography compared to $3 \%$ women with preeclampsia in the control group. Both for women with normal and abnormal coronary angiography we found about $6 \%$ to have had preeclampsia. The prevalence of preeclampsia overall in the Netherlands is estimated at 4\% [20] suggesting a two-fold increased risk of preeclampsia in our study group compared to the general Dutch population. This might suggest a higher prevalence of preeclampsia in our control group (normal angiography) than expected. A recall bias especially in the group of women who had a normal angiography might explain these differences. However, a recent reported study described that previous diagnosis of preeclampsia, eclampsia, or toxemia was verified with $80 \%$ sensitivity and $96 \%$ specificity 20 years post partum [21]. In addition, the controls had to undergo angiography for varying reasons, among these having multiple risk factors for developing CVD. Therefore as a group these women presumably had a higher for CVD than the general population [22].
In addition, a prospective study over several years will answer the question of this paper but evidence should be found earlier to prevent cardiovascular diseases in women. Recently, Hermes et al. [23], published a protocol stuyding modifiable cardiovascular risk factors in the course of 2.5 years after delivery by women with preeclampsia or hypertension during pregnancy. This might help in the determination during a longer period whether preeclampsia or hypertension during pregnancy is a long-standing risk factor or a stress test for later life-events.

In conclusion, we found that pregnancy complications including preeclampsia did not have an impact on cardiovascular disease in later life. Main contributors are hypercholesterolemia, tobacco use, obesity, and diabetes.

\section{REFERENCES}

[1] Graham, I., Atar, D., Borch-Johnsen, K., et al. (2008) European guidelines on CVD prevention in clinical practice: Executive summary. Giornale Italiano di Cardiologia Journal, 9, 11-59.

[2] Garovic, V.D. and Hayman, S.R. (2007) Hypertension in pregnancy: An emerging risk factor for CVD; nature clinical practice. Nature Clinical Practice Nephrology, 3, 613-622. doi:10.1038/ncpneph0623

[3] Harskamp, R.E. and Zeeman, G.G. (2007) Preeclamspia: At risk for remote cardiovascular disease. American Journal of the Medical Sciences, 334, 291-295. doi:10.1097/MAJ.0b013e3180a6f094

[4] Roberts, J.M. and Cooper, D.W. (2001) Pathogenesis and genetics of pre-eclampsia. Lancet, 357, 53-56. doi:10.1016/S0140-6736(00)03577-7

[5] Sattar, N. and Greer, I.A. (2002) Pregnancy complications and maternal cardiovascular risk: Opportunities for intervention and screening? British Medical Journal, 325, 157-160. doi:10.1136/bmj.325.7356.157

[6] Williams, D. (2003) Pregnancy: A stress test for life. Current Opinion in Obstetrics and Gynecology, 15, 465471. doi:10.1097/00001703-200312000-00002

[7] Agatisa, P.K., Ness, R.B., Roberts, J.M., Costantino, J.P., Kuller, L.H. and McLaughlin, M.K. (2004) Impairment of endothelial function in women with a history of preeclampsia: An indicator of cardiovascular risk. American Journal of Physiology-Heart and Circulatory Physiology, 286, H1389-H1393. doi:10.1152/ajpheart.00298.2003

[8] Berends, A.L., De Groot, C.J., Sijbrands, E.J., et al. (2008) Shared constitutional risks for maternal vascularrelated pregnancy complications and future cardiovascular disease. Hypertension, 51, 1034-1041. doi:10.1161/HYPERTENSIONAHA.107.101873

[9] Hubel, C.A., Snaedal, S., et al. (2000) Dyslipoproteinaemia in postmenopausal women with a history of eclampsia. BJOG, 107, 776-784. doi:10.1111/j.1471-0528.2000.tb13340.x

[10] Manten, G.T., Sikkema, M.J., Voorbij, H.A., Visser, G.H., 
Bruinse, H.W. and Franx, A. (2007) Risk factors for cardiovascular disease in women with a history of pregnancy complicated by preeclampsia or intrauterine growth restriction. Hypertens Pregnancy, 26, 39-50. doi:10.1080/10641950601146574

[11] Ness, R.B., Markovic, N., Bass, D., Harger, G. and Roberts, J.M. (2003) Family history of hypertension, heart disease, and stroke among women who develop hypertension in pregnancy. Obstetrics \& Gynecology, 102, 1366-1371. doi:10.1016/j.obstetgynecol.2003.08.011

[12] Blaauw, J., Van Pampus, M.G., et al. (2006) Increased intima-media thickness after early-onset preeclampsia. Obstetrics \& Gynecology, 107, 1345-1351. doi:10.1097/01.AOG.0000218097.22464.b4

[13] [No authors listed] (2000) Report of the National High Blood Pressure Education Program Working Group on high blood pressure in pregnancy. American Journal of Obstetrics \& Gynecology, 183, S1-S22.

[14] Sibai, B., Dekker, G. and Kupferminc, M. (2005) Preeclampsia. Lancet, 365, 785-799.

[15] Smith, G.C., Pell, J.P. and Walsh, D. (2001) Pregnancy complications and maternal risk of ischaemic heart disease: A retrospective cohort study of 129290 births. Lancet, 357, 2002-2006. doi:10.1016/S0140-6736(00)05112-6

[16] Hubel, C.A., Powers, R.W., Snaedal, S., et al. (2008) Creactive protein is elevated 30 years after eclamptic pregnancy. Hypertension, 51, 1499-1505. doi:10.1161/HYPERTENSIONAHA.108.109934

[17] Sibai, B.M., Ewell, M., et al. (1997) Risk factors associated with preeclampsia in healthy nulliparous women.
American Journal of Obstetrics \& Gynecology, 177, 1003-1010. doi:10.1016/S0002-9378(97)70004-8

[18] Valdés, G., Quezada, F., Marchant, E., et al. (2009) Association of remote hypertension in pregnancy with coronary artery disease: A case control study. Hypertension, 53, 733-738.

doi:10.1161/HYPERTENSIONAHA.108.127068

[19] Haukkamaa, L., Salminem, M., et al. (2004) Risk for subsequent coronary artery disease after preeclampsia. The American Journal of Cardiology, 93, 805-808. doi:10.1016/j.amjcard.2003.11.065

[20] Silva, L.M., Coolman, M., et al. (2008) Low socioeconomic status is a risk factor for preeclampsia: The generation R study. Journal of Hypertension, 26, 1200-1208. doi:10.1097/HJH.0b013e3282fcc36e

[21] Diehl, C.L., Brost, B.C., et al. (2008) Preeclampsia as a risk factor for cardiovascular disease later in life: Validation of a preeclampsia questionnaire. American Journal of Obstetrics \& Gynecology, 198, e11-e13. doi:10.1016/j.ajog.2007.09.038

[22] Melis, F., Vandenbrouke, J.P., Büller, H.R., et al. (2004) Estimates of risk of venous thrombosis during pregnancy and puerperium are not influenced by diagnostic suspicion and referral basis. American Journal of Obstetrics \& Gynecology, 191, 825-829. doi:10.1016/j.ajog.2004.02.004

[23] Hermes, W., Franx, A., Van Pampus, M.G., et al. (2009) 10-Year cardiovascular event risks for women who experienced hypertensive disorders in late pregnancy: The HyRAS study. BMC Pregnancy Childbirth, 10, 28. doi:10.1186/1471-2393-10-28 